\title{
Ultrasound-tailored functional hemispherectomy for surgical control of seizures in children
}

\author{
Paul M. Kanev, M.D., Catherine M. Foley, M.D., and Dan Miles, M.D.
}

Department of Neurosurgery, Henry Ford Hospital, Detroit, Michigan; Division of Child Neurology, Children's Hospital of Pittsburgh, University of Pittsburgh School of Medicine, Pittsburgh, Pennsylvania; and Department of Pediatrics and Comprehensive Epilepsy Center, Temple University School of Medicine and St. Christopher's Hospital for Children, Philadelphia, Pennsylvania

Functional hemispherectomy techniques have been designed to minimize the long-term complications of anatomical resection without reducing the effectiveness of seizure control. The authors have used an ultrasound-guided approach tailored to combine temporal lobectomy with frontal and occipital disconnections with a central topectomy of the lateral, insular, and interhemispheric cortex. This technique achieves a comprehensive functional disconnection and minimizes entrance and manipulation within the body of the lateral ventricle. Eight patients ranging in age from 10 months to 23 years with congenital paresis and medically intractable seizures underwent functional hemispherectomy via this technique. The average surgical time was 4.5 hours, and blood loss ranged from 90 to $400 \mathrm{ml}$. All but one patient was discharged after 5 days. Postoperative fever syndromes, aseptic meningitis, and infection were avoided. On long-term follow-up evaluation (range 18-60 months, mean 38 months), seven of eight patients remain seizure free and were not on a course of anticonvulsant agents. Advantages of this technique include avoiding entrance within the ventricle, a more predictable postoperative period, and reduced postoperative complications.

Key Words * functional hemispherectomy * epilepsy * porencephaly * intraoperative ultrasound

Many techniques have been used for the surgical control of epilepsy in children, ranging from focal resections and callosotomy to hemispherectomy. Regardless of age, nearly all patients have remained seizure free at long-term follow up following anatomical hemispherectomy.[2,9] Delayed complications of hemispherectomy, however, have included hydrocephalus, infection, bone flap loss, and hemorrhage. The most serious long-term sequela is superficial cerebral hemosiderosis $(\mathrm{SCH})$, which could lead to a resumption of seizures, progressive injury to the functioning cerebral hemisphere, or death.[3,7,8] During the last 20 years modified surgical techniques for hemispherectomy have been introduced in an attempt to minimize or eliminate these long-term complications without reducing the effectiveness of seizure control.[1,6,8-10,12] In 1983 Rasmussen[8] reported a functionally complete hemispherectomy procedure that preserved the occipital and frontal lobes but disconnected them from the central aspect of the cerebral hemispheres. A central cortical resection, callosotomy, and temporal lobectomy completed the procedure. Other modified hemispherectomy techniques have included hemidecortication and hemicorticectomy. With each successive modification there has been a steady movement toward 
maximum preservation of cortical tissue, exemplified by the recently reported periinsular hemispherotomy.[10]

Although modified hemispherectomy techniques appear to have eliminated $\mathrm{SCH}$, postoperative complications include hydrocephalus, infection, and aseptic meningitis, as well as fever and disconnection syndromes.[1,10] In children many of these complications may be further reduced by limiting entrance and manipulation within the lateral ventricle. We have used intraoperative ultrasound to minimize or avoid entrance into the ventricular system and combined the disconnections of the functional hemispherectomy technique with a central cortex topectomy and temporal lobectomy. We retrospectively analyzed our experience to evaluate the seizure control and complications of this surgical technique.

\section{CLINICAL MATERIAL AND METHODS}

\section{Patient Evaluation}

Patients with congenital hemiparesis and intractable seizures were referred to the Comprehensive Epilepsy Center at St. Christopher's Hospital for Children in Philadelphia where an evaluation was completed by a pediatric neurologist and a pediatric neurosurgeon. Multiple outpatient and videotelemetric electroencephalograms were obtained, and neuropsychological testing and intracarotid sodium amytal injection were performed in each patient older than age 5 years. Postoperatively, children were examined at 1 and 3 months and then followed every 6 months. Anticonvulsant medications were tapered and then withdrawn in patients who remained seizure free 1 year after surgery.

\section{Patient Population}

From 1991 to 1995,70 operations for the surgical treatment of epilepsy were performed in 60 patients. Of these patients, eight (four males and four females ranging in age from 10 months to 23 years) with congenital hemiparesis and intractable seizures underwent ultrasound-tailored functional hemispherectomy. The cerebral hemispheres were equally involved; the most common magnetic resonance (MR) imaging finding was porencephaly with an enlarged ipsilateral ventricle. Diffuse cerebral hemispheric architectural dysplasia was present in two patients with normal ventricle size. Sodium amytal carotid artery infusion of the affected cerebral hemisphere in four patients produced no transient deficits of language function or weakness of the contralateral lower extremity.

\section{Surgical Technique}

After induction of general anesthesia and bladder, arterial, and central venous line catheter placement, the patient is positioned prone with the head neutral and shoulders elevated. Shaving of the patient's hair is limited to the line of the Dandy incision. The scalp is prepared with povidone iodine scrub, alcohol, and povidone iodine solution. Nafcillin $(50 \mathrm{mg} / \mathrm{kg})$ and gentamicin $(1 \mathrm{mg} / \mathrm{kg})$ are infused prior to the skin incision and nafcillin is continuously administered for 36 hours postoperatively. In patients allergic to penicillin, $10 \mathrm{mg} / \mathrm{kg}$ vancomycin is substituted, which is infused slowly over a 1-hour period.

The temporalis muscle is divided along the path of the skin incision and elevated in a subperiosteal plane. A frontotemporoparietal bone flap is fashioned to within $0.5 \mathrm{~cm}$ of the sagittal plane and floor of the middle cranial fossa. The dura is opened along the perimeter of the exposure, reflected under tension toward the midline, and protected within moistened telfa.

A temporal lobectomy begins with an en bloc resection of the lateral temporal gyri, followed by 
ultrasound-guided entrance into the temporal horn and exposure of the hippocampus from the pes toward the atrium. The mesial temporal structures including the amygdala, hippocampus, and the uncus are removed using a subpial resection technique. The medial pia is left intact, protecting the cerebral peduncle, the third cranial nerve, and perforating and larger vessels about the circle of Willis.

The plane of occipital disconnection is identified with ultrasound; a pial incision is made in a coronal path along the lateral parietooccipital cortex through the atrium of the ventricle to the interhemispheric fissure, while avoiding or minimizing entrance into the ventricle. The deep white matter is divided using the ultrasonic aspirator, crossing behind the occipital horn, toward the interhemispheric fissure. The vein of Galen tributaries are visualized immediately posterior to the splenium of the corpus callosum. An intact interhemispheric pia ensures protection of these vessels and the calcarine and distal posterior cerebral arterial supply to the occipital lobe. Ultrasound is a useful gauge of the depth of disconnection as the interhemispheric fissure is approached. The central cerebral hemisphere is gently retracted to aid visualization (Fig. 1).

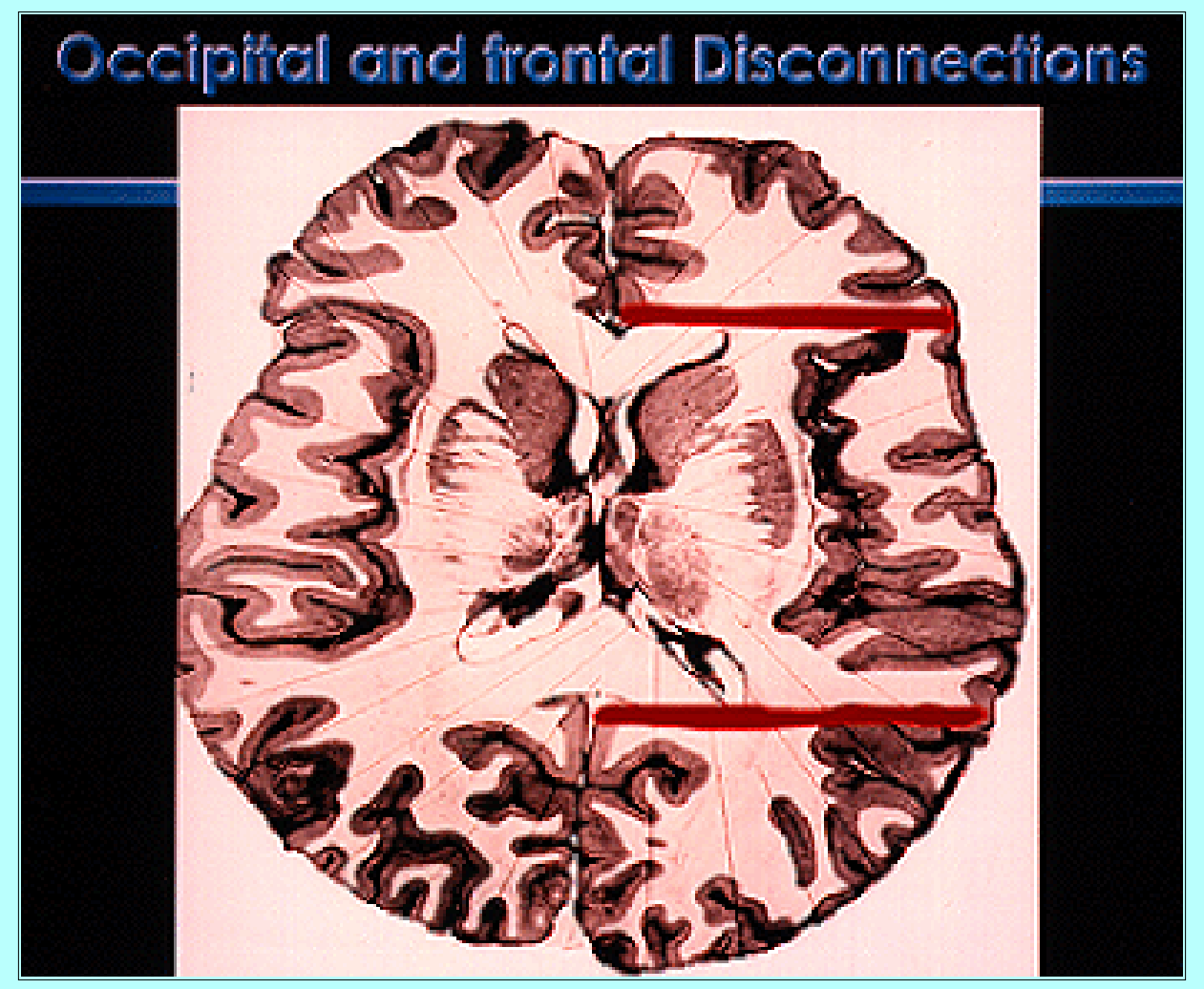

Fig. 1. Photograph showing how the posterior disconnection is guided by ultrasound to cross from the atrium of the ventricle along the lateral parietooccipital cortex toward the midline and interhemispheric fissure. The selected path minimizes entrance into the occipital horn of the lateral ventricle, divides the forceps major, and passes immediately posterior to the splenium of the corpus callosum. The frontal disconnection is guided by ultrasound to pass from the lateral frontal cortex toward the midline just anterior to the rostrum of the corpus callosum. The forceps minor is divided and entrance into the frontal horn of the lateral ventricle is avoided. (Modified from Roberts M, Hanaway J, Morest DK: Atlas of the Human Brain in Section, ed 2. Philadelphia: Lea \& Febiger, 1987, p 45.)

Using ultrasound, the coronal plane of the frontal disconnection is identified to pass in front of the rostrum of the corpus callosum, avoiding entrance into the frontal horn of the ventricle. A cortical incision is made adjacent to the lateral sphenoid wing and extends toward the midline. The white matter 
is divided using the ultrasonic aspirator, and through intact interhemispheric pia the anterior cerebral vessels are seen coursing about the rostrum of the corpus callosum. The internal frontal and frontopolar branches of the anterior cerebral artery are preserved (Fig. 1).

Central topectomy of the insular and opercular cortices involves subpial removal to adjacent sulcal margins, coagulating middle cerebral artery branches and extending across the lateral cerebral convexity toward the midline. The topectomy is continued along the falx until the pia of the sulcus of the corpus callosum is reached. Maintenance of the subpial plane protects numerous veins draining toward the sagittal sinus and the path of the distal pericallosal arteries (Fig. 2).

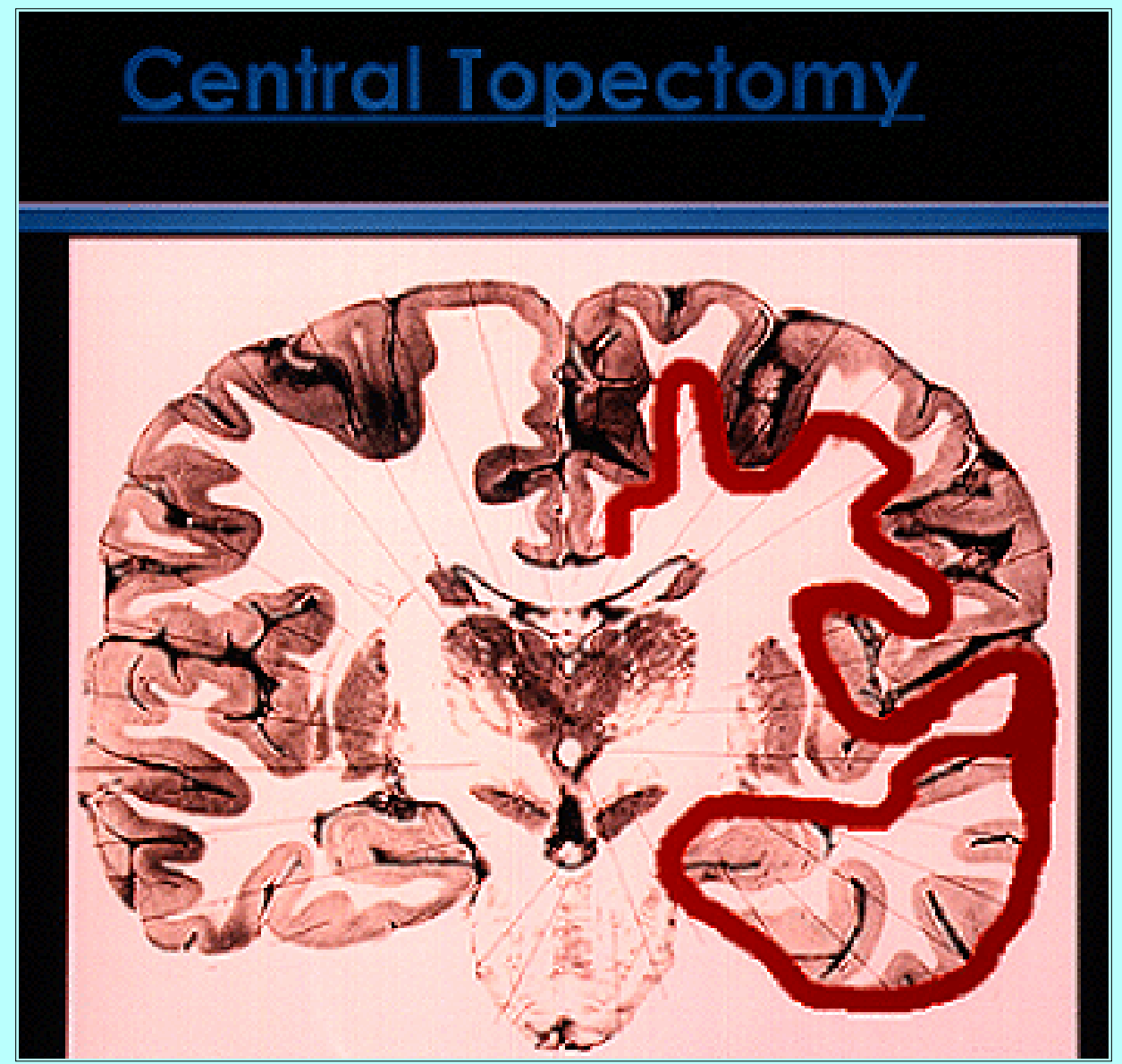

Fig. 2. Photograph showing how the topectomy of the central cortex begins at the sylvian fissure, coagulating distal middle cerebral artery branches, and extends toward the midline. The interhemispheric cortex is resected subpially until the sulcus of the corpus callosum is reached. Temporal lobectomy includes en bloc resection of the lateral cortex and subpial resection of mesial structures. (Modified from Roberts M, Hanaway J, Morest DK: Atlas of the Human Brain in Section, ed 2. Philadelphia: Lea \& Febiger, 1987, p 21.)

All resection margins are sparingly lined with Surgicel and the dura is sutured primarily with continuous running 4-0 vicryl, augmented with pericranial fascial grafts as required. The incision is closed in layers, without drains.

Arterial PCO2 is maintained at 28 to $30 \mathrm{~mm} \mathrm{Hg}$ until the temporal lobectomy and occipital and frontal disconnections are completed. The remainder of the procedure is completed with normal $\mathrm{PaCO} 2$. After induction of anesthesia, a $20-\mathrm{ml} / \mathrm{kg}$ intravenous saline infusion promotes mild hemodilution. Initial blood loss is supported with isotonic crystalloid solutions, and then replaced $\mathrm{ml}$ for $\mathrm{ml}$ with donor-directed 
packed red blood cells in each patient younger than age 20 years. Extubation is performed in the operating room prior to transfer to the pediatric intensive care unit for 24 to 36 hours of monitoring. Intravenously administered dexamethasone, $1 \mathrm{mg} / \mathrm{kg} / \mathrm{day}$, up to a maximum dose of $6 \mathrm{mg}$ every 6 hours, is continued for 3 days and discontinued without tapering. Oral intake is resumed on the 1st postoperative day, and the patient is encouraged to engage in out of bed activity on the 2 nd day. Physical therapists assist with postoperative activity and ambulation in each patient. A postoperative MR image is obtained in each patient before hospital discharge (Fig. 3).

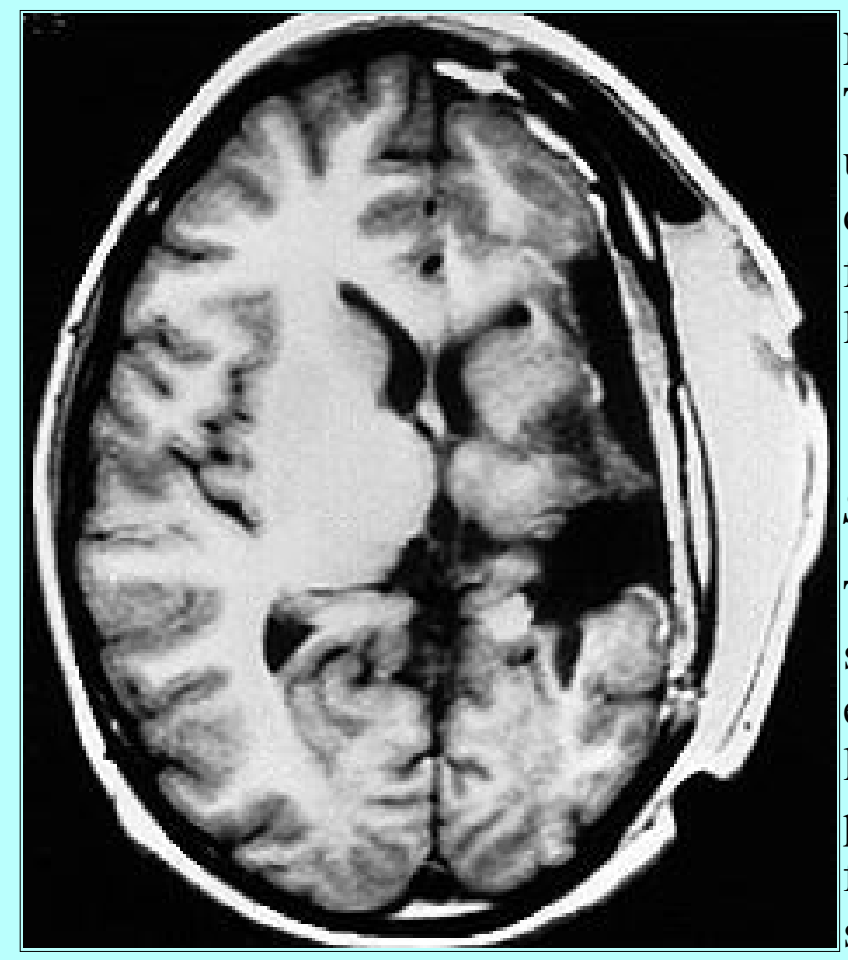

Fig. 3. Postoperative axial magnetic resonance

T1-weighted image, obtained 2 days after

ultrasound-guided functional hemispherectomy. There is considerable subcutaneous soft-tissue swelling. Note the frontal and occipital disconnections, extending from the lateral convexity to the interhemispheric fissure.

\section{RESULTS}

\section{Surgical Outcome}

The average length of surgery was 4.5 hours. There was no surgical mortality or complications due to anesthesia and no episodes of intraoperative hypotension or coagulopathy. Blood loss ranged from 90 to $400 \mathrm{ml}$ (mean $140 \mathrm{ml} ; 18 \mathrm{ml} / \mathrm{kg}$ in patients younger than age 4 years) and was replaced milliliter for milliliter with donor-directed packed red blood cells in seven of eight patients.

Postoperatively subcutaneous swelling was considerable but resolved in all patients within 14 days. Hemorrhage, fever, irritability, lethargy, infection, headaches, or cerebrospinal fluid leaks were not encountered. The first patient in our series was hospitalized for 14 days; subsequent patients were discharged on the 5 th postoperative day.

\section{Neurological Function}

There were no postoperative speech or language deficits. Immediately after surgery, a dense paresis occurred in the upper extremity in each patient. Weakness was greater in the hand and wrist than in the elbow or shoulder; however, all patients had antigravity shoulder, triceps, and biceps strength on discharge. Extension hand splints were constructed for nighttime restraint to minimize finger and wrist flexion contractures, and each patient was enrolled in outpatient physical and occupational therapy.

At 1-month follow-up evaluation, assist movements of the wrist had returned in six of eight patients, and full recovery of preoperative wrist and hand movements occurred in all patients by 4 months postoperatively. Increased long finger flexor tone persisted in three patients at 1-year follow up, requiring the long-term use of evening extension splints.

Contralateral leg strength was diminished in the immediate postoperative period and was barely antigravity in all patients. Tone and muscle strength improved rapidly, and all but one patient older than 1 year of age were ambulatory on discharge. The first patient in our series, an 8-year-old girl, required more intensive postoperative physical therapy than the other patients; this lengthened her hospitalization 
to 14 days. She was transferred to an inpatient pediatric rehabilitation institute and was ambulatory 27 days postoperatively.

\section{Long-Term Follow-Up Evaluation}

Seven of eight patients have remained seizure free during long-term follow-up study (range 18-60 months, mean 38 months). Preoperative anticonvulsant medications were continued for 1 year following surgery and then successfully withdrawn in each seizure-free patient. No long-term complication including hemorrhage, $\mathrm{SCH}$, infection, or skull defect was encountered. Four months postoperatively, headaches began in a 6-year-old boy; they increased in frequency and progressed to papilledema over the next 3 months. The headaches resolved after placement of a Delta Level I ventriculoperitoneal shunt. Complex partial seizures resumed 9 months postoperatively in this patient and combination anticonvulsant therapy was continued.

The quality of life improved postoperatively for patients and their families. School performance increased in the five patients who attended classes, and children were better integrated in their family and educational communities. Four children were able to participate in physical activities for the first time without helmet protection. The oldest patient began driving for the first time 18 months postoperatively and is currently employed as a carpenter's assistant.

\section{DISCUSSION}

\section{Complications of Hemispherectomy}

Superficial cerebral hemosiderosis is the most serious delayed complication of anatomical hemispherectomy. Shift and displacement of the intact cerebral hemisphere result in repetitive small hemorrhages into the large resection cavity with development of subdural membranes. There is progressive deposition of iron pigments on the contralateral cerebral hemisphere, ultimately leading to granular ependymitis, gliosis, new seizure activity, and death in up to $33 \%$ of patients.[4,7,8] Superficial cerebral hemosiderosis can occur up to 20 years after anatomical resection; the median onset is 8 years postoperatively.

Many functional hemispherectomy techniques have been designed to reduce or avoid delayed complications of anatomical resection while preserving excellent long-term seizure control. As described by Rasmussen,[8] the preserved frontal and occipital lobes support the contralateral hemisphere, minimizing displacement and shift that may be the source of repetitive small hemorrhages. Superficial cerebral hemosiderosis appears to be avoided by preservation of large polar blocks of cerebral tissue. More recent surgical techniques retain even larger elements of the cerebral cortex. Although reported follow-up study has been less than 10 years, delayed SCH and hemorrhagic complications have not been observed following other modified hemispherectomy surgical procedures.[10,12] There was no perioperative hemorrhage or evidence of SCH in our patients. The mean follow-up time is only 38 months: an interval too short to conclude that this long-term complication has been avoided with our technique.

Postoperative complications following modified hemispherectomy have included inflammatory meningitis and fever syndromes. Irritability, fever, lethargy, and diminished oral intake may commonly begin as early as the 2nd to 4th postoperative day, significantly prolonging hospitalization. Symptoms may improve with steroid use and typically resolve over 7 to 10 days. Villemure and Mascott[10] reported a series of patients with aseptic meningitis symptoms that occurred in each of 11 patients 
following periinsular hemispherotomy despite the maximum preservation of cortical tissue retained with their technique. In contrast to other modified hemispherectomy techniques, we have used intraoperative ultrasound to minimize entrance into the lateral ventricle. The resection of cortical tissue remains largely extraventricular, minimizing ependymal contact with Surgicel and blood degradation pigments. Postoperative fever syndromes were not encountered in our series, and all but the first child were discharged on the 5th postoperative day. This length of stay compares favorably to other series; patients were discharged 7 to 10 days following periinsular hemispherotomy,[10] whereas hospital stay was typically 15 to 20 days after classic functional hemispherectomy.[8]

\section{Anesthesia Considerations}

In contrast to the anesthesia techniques required for cerebral protection during craniotomies for tumor resection, anticipation of blood loss during modified hemispherectomy merits avoidance of hypovolemia. Mild, deliberate volume expansion with perioperative hemodilution $(20 \mathrm{ml} / \mathrm{kg})$ diminishes red blood cell loss from intraoperative bleeding and reduces the necessary volume of transfused packed red blood cells. Because these patients are otherwise in excellent general health with normal cardiac output, a hemoglobin count of $7.5 \mathrm{~g} / \mathrm{dl}$ can be tolerated before beginning transfusion. Intraoperative hematocrit analysis was possible within 90 seconds of blood sampling, matching transfusion volume to the actual replacement necessary. Critical attention to temperature control, use of humidified inspired air, and warming circuits for fluids and blood products was necessary to avoid coagulopathy and diminished platelet function.[5] A constant dialogue with the anesthesiologists during surgery minimized hypotension from delays in fluid replacement or transfusion. In contrast with other series, significant intraoperative bleeding in our patients was not encountered[1,4] and additional postoperative transfusion was not required.

\section{Analysis of Current Series}

Seven of eight patients in our series remain seizure free after withdrawal of anticonvulsant therapy. The remaining patient, a 6-year-old boy, experienced complex partial seizures after nearly 8 months of being free of seizures. The frequency and duration of his seizures remain considerably reduced compared with his preoperative state, and combination anticonvulsant therapy has been continued. Our experience compares favorably with the long-term seizure control reported by Winston, et al.[12] In their series, eight of 11 patients who underwent hemicorticectomy were seizure free and another two had near-complete seizure control. In another series reported recently by Carson, et al.,[2] long-term seizure control was achieved in $96 \%$ of 52 patients after hemidecortication. Three patients died in the perioperative period, and radiological evidence of hemorrhage within the resection cavity was detected in 21 patients. Seizure control was achieved in $78 \%$ of 32 patients who completed functional hemispherectomy at the Montreal Neurological Institute,[9] whereas nine (82\%) of 11 patients remained seizure free following periinsular hemispherotomy.[10]

Hydrocephalus leading to placement of a ventriculoperitoneal shunt occurred in a single patient (12.5\%). The complication of postoperative spinal fluid diversion is not unique to any single technique of hemispherectomy, nor can it be avoided by another technique. Hydrocephalus after anatomical hemispherectomy and hemidecortication has been reported in $20 \%$ to $50 \%$ of patients, $[1,11]$ whereas the need for postoperative shunting following modified hemispherectomy procedures was one in 11 children as reported by Winston, et al.,[12] and Villemure and Mascott.[10] The incidence of hydrocephalus appears to increase with more extensive cortical tissue resections. 


\section{CONCLUSIONS}

In our series the use of intraoperative ultrasound limited entrance and surgical manipulation in the body of the lateral ventricle during functional hemispherectomy. Central topectomy and polar cortical disconnection remained largely extraventricular and postoperative inflammatory meningitis syndromes were avoided. With perioperative hemodilution and critical hemostasis during surgery, transfusion was limited. We recommend this surgical technique as an alternative to the intraventricular resection of other modified hemispherectomy techniques. The procedure can be accomplished regardless of ventricle size and may be ideal for patients with hemimegalencephaly or Sturge-Weber syndrome, when an enlarged hemisphere may compromise cerebral retraction.

\section{Acknowledgment}

The authors are grateful for the counsel and many suggestions of Dr. George Ojemann, Professor of Neurological Surgery, University of Washington School of Medicine.

\section{References}

1. Carson BS, Javedan SP, Freeman JM, et al: Hemispherectomy: a hemidecortication approach and review of 52 cases. J Neurosurg 84:903-911, 1996

2. Davies KG, Maxwell RE, French LA: Hemispherectomy for intractable seizures: long-term results in 17 patients followed for up to 38 years. J Neurosurg 78:733-740, 1993

3. Falconer MA, Wilson PJ: Complications related to delayed hemorrhage after hemispherectomy. J Neurosurg 30:413-426, 1969

4. Griffiths PD, Welch RJ, Gardner-Medwin D, et al: The radiological features of hemimegalencephaly including three cases associated with proteus syndrome. Neuropediatrics 25:140-144, 1994

5. Haglund MM, Grady SM, Kanev PM, et al: Rapid infusion system for neurosurgical treatment of massive intraoperative hemorrhage. J Neurotrauma 11:623-627, 1994

6. Hoffman HJ, Hendrick EB, Dennis M, et al: Hemispherectomy for Sturge-Weber syndrome. Childs Brain 5:233-248, 1979

7. Oppenheimer DR, Griffith HB: Persistent intracranial bleeding as a complication of hemispherectomy. J Neurol Neurosurg Psychiat 9:229-240, 1966

8. Rasmussen T: Hemispherectomy for seizures revisited. Can J Neurol Sci 10:71-78, 1983

9. Villemure JG, Adams CBT, Hoffman HJ, et al: Hemispherectomy, in Engel J Jr (ed): Surgical Treatment of the Epilepsies, ed 2. New York: Raven Press, 1993, pp 511-518

10. Villemure JG, Mascott CR: Peri-insular hemispherotomy: surgical principles and anatomy. Neurosurgery 37:975-981, 1995

11. Vining EP, Freeman JM, Brandt J, et al: Progressive unilateral encephalopathy of childhood (Rasmussen's syndrome): a reappraisal. Epilepsia 34:639-650, 1993 
12. Winston KR, Welch K, Adler JR, et al: Cerebral hemicorticectomy for epilepsy. J Neurosurg 77:889-895, 1992

Manuscript received September 18, 1996.

Accepted in final form October 17, 1996.

Address reprint requests to: Paul M. Kanev, M.D., c/o Editorial Office, Department of Neurosurgery, Henry Ford Hospital, 2799 West Grand Boulevard, Detroit, Michigan 48202.

This review was presented at the annual meeting of the American Association of Neurological Surgeons, Minneapolis, Minnesota, April 27-May 2, 1996.

Click here to view Editor's Perspective. 